\title{
Eigenvalue estimates for submanifolds in Hadamard manifolds and product manifolds $N \times \mathbb{R}$
}

\author{
Jing MAO, Rongqiang Tu and Kai ZeNG \\ (Received June 4, 2018) \\ (Revised June 26, 2019)
}

\begin{abstract}
In this paper, we investigate submanifolds with locally bounded mean curvature in Hadamard manifolds, product manifolds $N \times \mathbb{R}$, submanifolds with bounded $\varphi$-mean curvature in the hyperbolic space, and successfully give lower bounds for the weighted fundamental tone and the first eigenvalue of the $p$-Laplacian.
\end{abstract}

\section{Introduction}

Let $(M, g)$ be an $n$-dimensional $(n \geq 2)$ smooth Riemannian manifold with the Riemannian metric $g$, the gradient operator $\nabla$ and the Laplacian $\Delta=\operatorname{div} \circ \nabla$. For an open bounded connected domain $\Omega \subset M$, the classical Dirichlet eigenvalue problem on $\Omega$ is actually to find possible real numbers $\lambda$ such that the boundary value problem (BVP for short)

$$
\begin{cases}\Delta u+\lambda u=0 & \text { in } \Omega, \\ u=0 & \text { on } \partial \Omega,\end{cases}
$$

has a nontrivial solution $u$. The desired real numbers $\lambda$ are called eigenvalues of $\Delta$, and the space of solutions of each $\lambda$ is called its eigenspace which is a vector space. It is well known that for the BVP (1), the self-adjoint operator $\Delta$ only has the discrete spectrum whose elements (i.e., eigenvalues) can be listed increasingly as follows

$$
0<\lambda_{1}(\Omega)<\lambda_{2}(\Omega) \leq \cdots \uparrow \infty,
$$

and each associated eigenspace has finite dimension. $\lambda_{i}(i \geq 1)$ is called the $i$ th Dirichlet eigenvalue of $\Delta$. By domain monotonicity of eigenvalues with

This work was supported in part by the NSF of China (Grant Nos. 11401131 and 11801496), the Fok Ying-Tung Education Foundation (China), and Key Laboratory of Applied Mathematics of Hubei Province (Hubei University).

2010 Mathematics Subject Classification. 53C40, 53C42, 58C40.

Key words and phrases. Eigenvalues; Drifting Laplacian; p-Laplacian; Hadamard manifolds; Product manifolds. 
vanishing Dirichlet data (cf. [4, pp. 17-18]), we know that $\lambda_{1}\left(\Omega_{1}\right) \leq \lambda_{1}\left(\Omega_{2}\right)$ if $\Omega_{1} \supset \Omega_{2}$.

For a domain $\Omega \subseteq M$ (with or without boundary $\partial \Omega$ ), one can define the fundamental tone $\lambda_{1}^{*}(\Omega)$ of $\Omega$ as

$$
\lambda_{1}^{*}(\Omega):=\inf \left\{\frac{\int_{\Omega}\|\nabla f\|^{2} d v}{\int_{\Omega} f^{2} d v} \mid f \in W_{0}^{1,2}(\Omega), f \neq 0\right\},
$$

where $W_{0}^{1,2}(\Omega)$ is the completion of the set $C_{0}^{\infty}(\Omega)$ of smooth functions compactly supported on $\Omega$ under the Sobolev norm $\|u\|_{1,2}=\left\{\int_{\Omega}\left(|u|^{2}+\right.\right.$ $\left.\left.\|\nabla u\|^{2}\right) d v\right\}^{1 / 2}$, with $d v$ the Riemannian volume element with respect to the metric $g$. In what follows, without specification, $\|\cdot\|$ denotes the norm of some prescribed vector field, and, for the sake of simplicity, the measure dv will be omitted from integrals. If $\Omega$ is unbounded, then the fundamental tone $\lambda_{1}^{*}(\Omega)$ coincides with the infimum $\inf (\Sigma)$ of the spectrum $\Sigma \subseteq[0,+\infty)$ of the unique self-adjoint extension of the Laplacian $\Delta$ acting on $C_{0}^{\infty}(\Omega)$, which is also denoted by $\Delta$. If $\Omega$ has compact closure and piecewise smooth boundary $\partial \Omega$ (maybe nonempty), $\lambda_{1}^{*}(\Omega)$ equals the first closed eigenvalue (if $\partial \Omega=\varnothing$ ) or the first Dirichlet eigenvalue (if $\partial \Omega \neq \varnothing$ ) $\lambda_{1}(\Omega)$ of $\Delta$. If $\Omega_{1} \subset \Omega_{2}$ are bounded domains, then $\lambda_{1}^{*}\left(\Omega_{1}\right) \geq \lambda_{1}^{*}\left(\Omega_{2}\right) \geq 0$.

From the above introduction, we know that for a bounded domain $\Omega$ with boundary, the degree of smoothness of the boundary $\partial \Omega$ decides the fundamental tone $\lambda_{1}^{*}(\Omega)$ would degenerate into the first Dirichlet eigenvalue $\lambda_{1}(\Omega)$ of the Laplacian or not.

Let $B_{M}(q, \ell)$ be a geodesic ball, with center $q$ and radius $\ell$, on a complete noncompact Riemannian manifold $M$. By the monotonicity of the first Dirichlet eigenvalue $\lambda_{1}$ or the fundamental tone $\lambda_{1}^{*}$, one can define a limit $\lambda_{1}(M)$ by

$$
\lambda_{1}(M):=\lim _{\ell \rightarrow \infty} \lambda_{1}\left(B_{M}(q, \ell)\right)=\lim _{\ell \rightarrow \infty} \lambda_{1}^{*}\left(B_{M}(q, \ell)\right)
$$

which is independent of the choice of the center $q$. Clearly, $\lambda_{1}(M) \geq 0$. Schoen and Yau [18, p. 106] suggested that it is an important question to find conditions which will imply $\lambda_{1}(M)>0$. Speaking in other words, manifolds with $\lambda_{1}(M)>0$ might have some special geometric properties. There are many interesting results supporting this. For instance, Mckean [17] showed that for an $n$-dimensional complete noncompact, simply connected Riemannian manifold $M$ with sectional curvature $K_{M} \leq-a^{2}<0, \lambda_{1}(M) \geq \frac{(n-1)^{2} a^{2}}{4}>0$, and moreover, $\lambda_{1}\left(\mathbb{H}^{n}\left(-a^{2}\right)\right)=\frac{(n-1)^{2} a^{2}}{4}$ with $\mathbb{H}^{n}\left(-a^{2}\right)$ the $n$-dimensional hyperbolic space of sectional curvature $-a^{2}$. Grigor'yan [11] showed that if $\lambda_{1}(M)>0$, then $M$ is non-parabolic, i.e., there exists a non-constant bounded subharmonic function on $M$. Cheung and Leung [6] proved that if $M$ is an $n$-dimensional 
complete minimal submanifold in the hyperbolic $m$-space $\mathbb{H}^{m}(-1)$, then $\lambda_{1}(M) \geq \frac{(n-1)^{2}}{4}>0$, and moreover, $M$ is non-parabolic. They also showed that if furthermore $M$ has at least two ends, then there exists a non-constant bounded harmonic function on $M$ with finite Dirichlet energy.

Consider the BVP

$$
\begin{cases}\Delta_{\varphi} u+\lambda u=0 & \text { in } \Omega, \\ u=0 & \text { on } \partial \Omega,\end{cases}
$$

where $\Omega \subset M$ is an open bounded connected domain in a given Riemannian manifold $M, \Delta_{\varphi} u:=\Delta u-\langle\nabla u, \nabla \varphi\rangle$ is the weighted Laplacian (also called the drifting Laplacian) on $M$, and $\varphi$ is a real-valued smooth function on $M$. Similar to the BVP (1), $\Delta_{\varphi}$ in the BVP (2) only has the discrete spectrum and all the eigenvalues in the discrete spectrum can be listed increasingly. By Rayleigh's theorem and the max-min principle, it is easy to know that the first Dirichlet eigenvalue $\lambda_{1, \varphi}(\Omega)$ of $\Delta_{\varphi}$ on $\Omega$ can be characterized by

$$
\lambda_{1, \varphi}(\Omega)=\inf \left\{\frac{\int_{\Omega}\|\nabla f\|^{2} e^{-\varphi}}{\int_{\Omega} f^{2} e^{-\varphi}} \mid f \in W_{0}^{1,2}(\Omega), f \neq 0\right\} .
$$

Similar to the case of the Laplacian, for a (bounded or unbounded) domain $\Omega \subseteq M$ (with or without boundary $\partial \Omega$ ), one can define the weighted fundamental tone $\lambda_{1, \varphi}^{*}(\Omega)$ of $\Omega$ as

$$
\lambda_{1, \varphi}^{*}(\Omega):=\inf \left\{\frac{\int_{\Omega}\|\nabla f\|^{2} e^{-\varphi}}{\int_{\Omega} f^{2} e^{-\varphi}} \mid f \in W_{0}^{1,2}(\Omega), f \neq 0\right\},
$$

and it is not difficult to get that $\lambda_{1, \varphi}^{*}(\Omega)=\lambda_{1, \varphi}(\Omega)$ if $\Omega$ has compact closure and its boundary $\partial \Omega$ is piecewise smooth.

Domain monotonicity of eigenvalues with vanishing Dirichlet data also holds for the first Dirichlet eigenvalue of $\Delta_{\varphi}$ (see, e.g., [8, Lemma 1.5]). This implies that for a complete noncompact Riemannian manifold $M$, one can define the limit

$$
\lambda_{1, \varphi}(M):=\lim _{\ell \rightarrow \infty} \lambda_{1, \varphi}\left(B_{M}(q, \ell)\right)=\lim _{\ell \rightarrow \infty} \lambda_{1, \varphi}^{*}\left(B_{M}(q, \ell)\right),
$$

which is independent of the choice of the point $q$ and can be seen as a generalization of $\lambda_{1}(M)$. Clearly, $\lambda_{1, \varphi}(M) \geq 0$ and if $\varphi=$ const., then $\lambda_{1, \varphi}(M)=$ $\lambda_{1}(M)$. Based on Schoen-Yau's suggestion mentioned before, it is natural to ask:

QUESTION 1. For a given complete noncompact Riemannian manifold $M$, under what conditions, $\lambda_{1, \varphi}(M)>0$ ? 
For an $n$-dimensional $(n \geq 2)$ complete noncompact submanifold of a hyperbolic space whose norm of the mean curvature vector $\|H\|$ satisfies $\|H\| \leq \alpha<n-1$, Du and Mao [8, Theorem 1.7] proved that if $\|\varphi\| \leq C^{1}$, then $\lambda_{1, \varphi}(M) \geq \frac{(n-1-\alpha-C)^{2}}{4}$, with equality attained when $M$ is totally geodesic and $\varphi=$ const., which generalized Cheung-Leung's and Mckean's conclusions mentioned before.

Consider the BVP

$$
\begin{cases}\Delta_{p} u+\lambda|u|^{p-2} u=0 & \text { in } \Omega, \\ u=0 & \text { on } \partial \Omega,\end{cases}
$$

where $\Omega \subset M$ is an open bounded connected domain in a given Riemannian manifold $M, \Delta_{p} u:=\operatorname{div}\left(\|\nabla u\|^{p-2} \nabla u\right)$ is the nonlinear $p$-Laplacian of $u$ with $1<p<\infty$. It is known that (3) has a positive weak solution, which is unique modulo the scaling, in $W_{0}^{1, p}(\Omega)$, the completion of the set $C_{0}^{\infty}(\Omega)$ of smooth functions compactly supported on $\Omega$ under the Sobolev norm $\|u\|_{1, p}=$ $\left\{\int_{\Omega}\left(|u|^{p}+\|\nabla u\|^{p}\right)\right\}^{1 / p}$, and the first Dirichlet eigenvalue $\lambda_{1, p}(\Omega)$ of the $p$-Laplacian in the eigenvalue problem (3) can be characterized by

$$
\lambda_{1, p}(\Omega)=\inf \left\{\frac{\int_{\Omega}\|\nabla f\|^{p}}{\int_{\Omega}|f|^{p}} \mid f \in W_{0}^{1, p}(\Omega), f \neq 0\right\} .
$$

The (closed or Dirichlet) eigenvalue problem of the $p$-Laplacian has been studied by the first named author and some interesting conclusions have been obtained (see, e.g., [7, 8, 13, 14]). Domain monotonicity of eigenvalues with vanishing Dirichlet data also holds for the first Dirichlet eigenvalue of $\Delta_{p}$ (see, e.g., [8, Lemma 1.1]). This implies that for a complete noncompact Riemannian manifold $M$, one can define the limit

$$
\lambda_{1, p}(M):=\lim _{\ell \rightarrow \infty} \lambda_{1, p}\left(B_{M}(q, \ell)\right)
$$

which is independent of the choice of the point $q$ and can be seen as a generalization of $\lambda_{1}(M)$. Clearly, $\lambda_{1, p}(M) \geq 0$ and if $p=2$, then $\lambda_{1, p}(M)=$ $\lambda_{1}(M)$. Based on Schoen-Yau's suggestion mentioned before, it is natural to ask:

QUESTION 2. For a given complete noncompact Riemannian manifold $M$, under what conditions, $\lambda_{1, p}(M)>0$ ?

\footnotetext{
${ }^{1}$ It is easy to know that the constant $C$ satisfies $C<n-1-\alpha$, which is the potential assumption in [8, Theorem 1.7], since in the proof of [8, Theorem 1.7], the positive number $\varepsilon$ is chosen to be $\varepsilon=(n-1-\alpha-C) / 2$.
} 
For an $n$-dimensional $(n \geq 2)$ complete noncompact submanifold of a hyperbolic space whose norm of the mean curvature vector $\|H\|$ satisfies $\|H\| \leq$ $\alpha<n-1$, Du and Mao [8, Theorem 1.3] proved $\lambda_{1, p}(M) \geq\left(\frac{n-1-\alpha}{p}\right)^{p}>0$, with equality attained when $M$ is totally geodesic and $p=2$, which generalized Cheung-Leung's and Mckean's conclusions mentioned before.

The purpose of this paper is trying to positively answer Questions 1 and 2 further. In fact, we have obtained the following facts:

- By introducing a quantity $c(\Omega)$ for a domain $\Omega$ with compact closure (see Definition 1), Bessa-Montenegro type lower bounds for the weighted fundamental tone $\lambda_{1, \varphi}^{*}(\Omega)$ and the first eigenvalue $\lambda_{1, p}(\Omega)$ of the $p$-Laplacian can be obtained-see Lemma 1. By applying the Hessian comparison theorem, domain monotonicity of eigenvalues with vanishing Dirichlet data for $\lambda_{1, \varphi}^{*}(\cdot)$ and $\lambda_{1, p}(\cdot)$, Bessa-Montenegro type lower bounds would give us Mckean-type lower bounds for Hadamard manifolds with strictly negative sectional curvature-see Lemma 2.

- Let $\phi: M \rightarrow Q$ be an isometric immersion from $n$-dimensional $(n \geq 2)$ Riemannian manifold to an $m$-dimensional Riemannian manifold, and moreover, $M$ has locally bounded mean curvature (see Definition 2). For any connected component $\Omega$ of $\phi^{-1}\left(\overline{B_{Q}(q, r)}\right)$ with $q \in Q \backslash \phi(M)$, and $r>0$, under different assumptions on sectional curvatures, some strictly positive lower bounds have been obtained for the weighted fundamental tone $\lambda_{1, \varphi}^{*}(\Omega)$ (no matter $\Omega$ is bounded or unbounded) and the first eigenvalue $\lambda_{1, p}(\Omega)$ of the $p$-Laplacian (in this case, $\Omega$ is bounded and has piecewise smooth boundary)—see Theorem 2. As a direct consequence, if furthermore $M$ is noncompact with bounded mean curvature (stronger than the locally bounded mean curvature assumption) and the sectional curvature of $Q$ is bounded from above by some strictly negative constant, then $\lambda_{1, \varphi}(M)$ and $\lambda_{1, p}(M)$ have strictly positive lower bounds - see Corollary 4.

- Recently, because of the discovery of many interesting examples of minimal surfaces in product spaces $N \times \mathbb{R}$ (see, e.g., $[15,16]$ ), the study of this kind of spaces has attracted geometers' attention. Based on this, we investigate submanifolds $\Omega$, with locally bounded mean curvature, of $N \times \mathbb{R}$ and would like to know "under what conditions, $\lambda_{1, \varphi}^{*}(\Omega)>0$ and $\lambda_{1, p}(\Omega)>0$ ?". A positive answer has been given-see Theorem 3 for details.

- For an $n$-dimensional $(n \geq 2)$ complete non-compact $\varphi$-minimal submanifold $M$ of the weighted manifold $\left(\mathbb{H}^{m}(-1), e^{-\varphi} d v\right)$, where $\mathbb{H}^{m}(-1)$ is the hyperbolic $m$-space with sectional curvature $-1, \varphi$ is a real-valued smooth function on $\mathbb{H}^{m}(-1)$ and $d v$ is the volume element, a strictly 
positive lower bound has been obtained for the first eigenvalue $\lambda_{1, p}(M)$ for the $p$-Laplacian on $M$-see Theorem 4 for details.

- Interesting new lower bounds for the first Dirichlet eigenvalues of the weighted Laplacian and the $p$-Laplacian on geodesic balls of complete Riemannian manifolds have been given - see Theorem 5 for details.

\section{Bessa-Montenegro type and Mckean-type lower bounds for the weighted fundamental tone and the first eigenvalue of the $p$-Laplacian}

By using a notion introduced in [1], we can give lower bounds for the weighted fundamental tone for arbitrary bounded domains, and the lowest eigenvalue for the Dirichlet eigenvalue problem of the weighted Laplacian and the $p$-Laplacian on normal domains.

Definition 1 ([1]). Let $\Omega \subset M$ be a domain with compact closure in a $C^{\infty}$ Riemannian manifold $M$. Let $\mathscr{X}(\Omega)$ be the set of all smooth vector fields $X$ on $\Omega$ with $\|X\|_{\infty}:=\sup _{\Omega}\|X\|<\infty$ and inf $\operatorname{div} X>0$ with div the divergence operator on $M$. Define $c(\Omega)$ by

$$
c(\Omega):=\sup \left\{\frac{\inf \operatorname{div} X}{\|X\|_{\infty}}: X \in \mathscr{X}(\Omega)\right\} .
$$

REMARK 1. As shown in [1, Remark 2.2], it is easy to get that $\mathscr{X}(\Omega)$ is not empty. This is because the boundary value problem (BVP for short)

$$
\begin{cases}\Delta u=1, & \text { in } \Omega \\ u=0, & \text { on } \partial \Omega\end{cases}
$$

always has a solution on a bounded domain $\Omega \subset M$, and then at least one can choose $X=\nabla u$, the gradient of $u$, which implies that $\operatorname{div}(X)=1$ and $\|X\|_{\infty}<\infty$.

Now, we can prove the following.

LeMma 1. Let $\Omega \subset M$ be a domain with compact closure and nonempty boundary (i.e., $\partial \Omega \neq \varnothing$ ) in a Riemannian manifold $M$. Then we have

$$
\lambda_{1, \varphi}^{*}(\Omega) \geq \frac{\left(c(\Omega)-c^{+}\right)^{2}}{4}>0
$$

provided $\|\nabla \varphi\| \leq c^{+}<c(\Omega)$, where $c^{+}$is the supremum of the norm of the gradient of $\varphi$ and is strictly less than $c(\Omega)$, and $c(\Omega)$ is given by (4). Moreover, if furthermore the boundary $\partial \Omega$ is piecewise smooth, then we have

$$
\lambda_{1, p}(\Omega) \geq\left(\frac{c(\Omega)}{p}\right)^{p}>0 .
$$


Proof. Taking $f \in C_{0}^{\infty}(\Omega)$, the set of all smooth functions compactly supported on $\Omega$, and $X \in \mathscr{X}(\Omega)$. By a direct calculation, we have

$$
\begin{aligned}
\operatorname{div}\left(|f|^{p} X\right) & =\left\langle\nabla|f|^{p}, X\right\rangle+|f|^{p} \operatorname{div} X \\
& \geq-p|f|^{p-1}\|\nabla f\| \sup \|X\|+\inf \operatorname{div} X \cdot|f|^{p} .
\end{aligned}
$$

By Young's inequality, one can obtain

$$
|f|^{p-1}\|\nabla f\|=\varepsilon|f|^{p-1} \cdot \frac{\|\nabla f\|}{\varepsilon} \leq \frac{\left(\frac{\|\nabla f\|}{\varepsilon}\right)^{p}}{p}+\frac{\left(\varepsilon|f|^{p-1}\right)^{p /(p-1)}}{\frac{p}{p-1}},
$$

where $\varepsilon>0$ is a parameter determined later. Substituting the above inequality into (5) yields

$$
\operatorname{div}\left(|f|^{p} X\right) \geq-p \sup \|X\|\left[\frac{\left(\frac{\|\nabla f\|}{\varepsilon}\right)^{p}}{p}+\frac{\left(\varepsilon|f|^{p-1}\right)^{p /(p-1)}}{\frac{p}{p-1}}\right]+\inf \operatorname{div} X \cdot|f|^{p} .
$$

Choosing

$$
\varepsilon=\left(\frac{\inf \operatorname{div} X}{p \sup \|X\|}\right)^{(p-1) / p},
$$

in (6), integrating both sides of (6) over $\Omega$ and using the divergence theorem, we have

$$
\int_{\Omega}\|\nabla f\|^{p} \geq\left(\frac{\inf \operatorname{div} X}{p \sup \|X\|}\right)^{p} \int_{\Omega}|f|^{p}
$$

which implies

$$
\lambda_{1, p}(\Omega) \geq\left(\frac{c(\Omega)}{p}\right)^{p}
$$

by taking the supremum over all vector fields $X \in \mathscr{X}(\Omega)$ to the RHS of (7).

If $\|\nabla \varphi\| \leq c^{+}<c(\Omega)$ with $c^{+} \geq 0$ the supremum of $\|\nabla \varphi\|$, then we have

$$
\begin{gathered}
\operatorname{div}\left(f^{2} X e^{-\varphi}\right)=e^{-\varphi}\left\langle\nabla f^{2}, X\right\rangle+f^{2} e^{-\varphi} \operatorname{div} X-f^{2} e^{-\varphi}\langle\nabla \varphi, X\rangle \\
\geq e^{-\varphi}\left[-2|f| \cdot\|\nabla f\| \cdot \sup \|X\|+f^{2} \inf \operatorname{div} X-f^{2} c^{+} \sup \|X\|\right] \\
\geq e^{-\varphi}\left[\left(-\varepsilon f^{2}-\frac{\|\nabla f\|^{2}}{\varepsilon}\right) \sup \|X\|+f^{2} \inf \operatorname{div} X\right. \\
\left.-f^{2} c^{+} \sup \|X\|\right]
\end{gathered}
$$


where $\varepsilon>0$ is a parameter determined later. Integrating both sides of (8) and using the divergence theorem, we have

$$
\int_{\Omega}\|\nabla f\|^{2} e^{-\varphi} \geq \frac{\varepsilon\left(\inf \operatorname{div} X-c^{+} \sup \|X\|-\varepsilon \sup \|X\|\right)}{\sup \|X\|} \int_{\Omega} f^{2} e^{-\varphi} .
$$

On the other hand, since

$$
\frac{\varepsilon\left(\inf \operatorname{div} X-c^{+} \sup \|X\|-\varepsilon \sup \|X\|\right)}{\sup \|X\|} \leq\left(\frac{\frac{\inf \operatorname{div} X}{\sup \|X\|}-c^{+}}{2}\right)^{2}
$$

with equality holds if and only if $\varepsilon=\frac{\inf \operatorname{div} X}{2 \sup \|X\|}-\frac{c^{+}}{2}>0$, we can obtain

$$
\lambda_{1, \varphi}(\Omega) \geq \frac{\left(c(\Omega)-c^{+}\right)^{2}}{4}>0
$$

by choosing $\varepsilon=\frac{\inf \operatorname{div} X}{2 \sup \|X\|}-\frac{c^{+}}{2}$ in (9) and by taking the supremum over all vector fields $X \in \mathscr{X}(\Omega)$. This completes the proof of Lemma 1 .

REMARK 2. (1) Clearly, when $p=2$ (or $\varphi=$ const.), the nonlinear $p$-Laplacian (or the weighted Laplacian) degenerate into the Laplacian. Correspondingly, $\lambda_{1, p}(\Omega)=\lambda_{1}^{*}(\Omega)$ (or $\lambda_{1, \varphi}(\Omega)=\lambda_{1}^{*}(\Omega), c^{+}=0$ ), and moreover, $\lambda_{1}^{*}(\Omega) \geq\left(\frac{c(\Omega)}{2}\right)^{2}$, which is the lower bound for $\lambda_{1}^{*}(\Omega)$ in [1, Lemma 2.3] given by Bessa and Montenegro. Based on this fact, we would like to use BessaMontenegro type lower bounds to call the lower bounds for the lowest Dirichlet eigenvalue (resp., the weighted fundamental tone) shown in Lemma 1. Besides, to prove Bessa-Montenegro type lower bounds here, we only need to consider vector fields smooth almost every in $\Omega$ such that $\int_{\Omega} \operatorname{div}\left(|f|^{p} X\right)=0$ or $\int_{\Omega} \operatorname{div}\left(f^{2} X e^{-\varphi}\right)=0$ for all $f \in C_{0}^{\infty}(\Omega)$.

(2) It has been shown in [1, Remark 2.7] that $c(\Omega) \leq h(\Omega)$ with $h(\Omega):=$ $\inf _{A \subset \Omega} \frac{\operatorname{vol}(\partial A)}{\operatorname{vol}(A)}$ the Cheeger's constant. However, in some cases, for instance, for balls in the Euclidean space or Hadamard manifolds, $c(\Omega)=h(\Omega)$. The advantage of defining $c(\Omega)$ is the computability of lower bounds for $\lambda_{1, p}(\Omega)$, $\lambda_{1, \varphi}(\Omega)$ via any lower bound for $c(\Omega)$, and this way can be applied to arbitrary domains. Besides, we can use Lemma 1 to derive Mckean-type lower bounds below-see Lemma 2 for details.

Applying Lemma 1, one can get the following conclusion directly.

Corollary 1. Let $\Omega \subset M$ be a normal domain with compact closure in a smooth Riemannian manifold $M$. For the BVP

$$
\begin{cases}\Delta v=1, & \text { in } \Omega, \\ v=0, & \text { on } \partial \Omega\end{cases}
$$


we have

$$
\lambda_{1, p}(\Omega) \geq\left(\frac{1}{p\|\nabla v\|_{\infty}}\right)^{p}>0
$$

Besides,

$$
\lambda_{1, \varphi}^{*}(\Omega) \geq \frac{\left(\frac{1}{\|\nabla v\|_{\infty}}-c^{+}\right)^{2}}{4}>0
$$

provided $\|\nabla \varphi\| \leq c^{+}<\frac{1}{\|\nabla v\|_{\infty}}$, where $c^{+}$is the supremum of the norm of the gradient of $\varphi$ and is strictly less than $\frac{1}{\|\nabla v\|_{\infty}}$.

COROLlary 2. There are no smooth bounded vector fields $X: M \rightarrow$ $T M$ with $\inf _{M} \operatorname{div} X>0$ on complete noncompact manifolds $M$ such that $\lambda_{1, p}(M)=0, \lambda_{1, \varphi}(M)=0$. In particular, there is no such vector field on $\mathbb{R}^{n}$.

As an interesting application of Lemma 1, we can obtain Mckean-type lower bounds for the first eigenvalues of the drifting Laplacian and the $p$-Laplacian on the prescribed Hadamard manifold. However, in order to prove that, we need to use the Hessian comparison theorem below.

THEOREM 1 (Hessian comparison theorem). Let $M$ be a complete Riemannian manifold and $x_{0}, x \in M$. Let $\gamma:[0, \rho(x)] \rightarrow M$ be a minimizing geodesic joining $x_{0}$ and $x$, where $\rho(x)$ is the distance function $\operatorname{dist}_{M}\left(x_{0}, x\right)$. Let $K$ be the sectional curvature of $M$ and $\mu_{i}(\rho), i=0,1$, be functions defined by

$$
\mu_{0}(\rho)= \begin{cases}k_{0} \operatorname{coth}\left(k_{0} \rho(x)\right), & \text { if } \inf _{\gamma} K=-k_{0}^{2}, \\ \frac{1}{\rho(x)}, & \text { if } \inf _{\gamma} K=0, \\ k_{0} \cot \left(k_{0} \rho(x)\right), & \text { if } \inf _{\gamma} K=k_{0}^{2} \text { and } \rho<\frac{\pi}{2 k_{0}}\end{cases}
$$

and

$$
\mu_{1}(\rho)= \begin{cases}k_{1} \operatorname{coth}\left(k_{1} \rho(x)\right), & \text { if } \sup _{\gamma} K=-k_{1}^{2}, \\ \frac{1}{\rho(x)}, & \text { if } \sup _{\gamma} K=0, \\ k_{1} \cot \left(k_{1} \rho(x)\right), & \text { if } \sup _{\gamma} K=k_{1}^{2} \text { and } \rho<\frac{\pi}{2 k_{1}} .\end{cases}
$$

Then the Hessians of $\rho$ and $\rho^{2}$ satisfy

$$
\begin{aligned}
& \mu_{1}(\rho(x)) \cdot\|X\|^{2} \leq \operatorname{Hess} \rho(x)(X, X) \leq \mu_{0}(\rho(x)) \cdot\|X\|^{2}, \\
& \text { Hess } \rho(x)\left(\gamma^{\prime}, \gamma^{\prime}\right)=0,
\end{aligned}
$$


$2 \rho(x) \cdot \mu_{1}(\rho(x)) \cdot\|X\|^{2} \leq \operatorname{Hess} \rho^{2}(x)(X, X) \leq 2 \rho(x) \cdot \mu_{0}(\rho(x)) \cdot\|X\|^{2}$,

Hess $\rho^{2}(x)\left(\gamma^{\prime}, \gamma^{\prime}\right)=2$,

where $X$ is any vector in $T_{x} M$ perpendicular to $\gamma^{\prime}(\rho(x))$.

Hence, by applying Theorem 1, for the distance function $\rho(x)$ on an $n$-dimensional Riemannian manifold $M$, we can get

$$
2(n-1) \rho(x) \mu_{1}(\rho(x))+2 \leq \Delta \rho^{2}(x) \leq 2(n-1) \rho(x) \mu_{0}(\rho(x))+2 .
$$

LeMma 2. Let $M$ be an $n$-dimensional $(n \geq 2)$ Hadamard manifold whose sectional curvature satisfies $K_{M} \leq-a^{2}<0, a>0$. Then we have

$$
\lambda_{1, p}(M) \geq\left[\frac{(n-1) \cdot a}{p}\right]^{p}>0 .
$$

Moreover,

$$
\lambda_{1, \varphi}(M) \geq\left[\frac{(n-1) \cdot a-c^{+}}{2}\right]^{2}>0
$$

provided $\|\nabla \varphi\| \leq c^{+}<(n-1) a$, where $c^{+}$is the supremum of the norm of the gradient of $\varphi$ and is strictly less than $(n-1) a$.

Proof. Let $\rho: M \rightarrow \mathbb{R}$ be the distance function to a point $p \in M \backslash \Omega$ with $\Omega$ a normal domain in $M$, and let $X=\nabla \rho$. By (10), we have

$$
\Delta \rho(x)=\operatorname{div} X \geq(n-1) \cdot a \cdot \operatorname{coth}(a \cdot \rho(x)) \geq(n-1) \cdot a .
$$

By Lemma 1, it follows that

$$
\lambda_{1, p}(\Omega) \geq\left[\frac{(n-1) \cdot a}{p}\right]^{p}
$$

and

$$
\lambda_{1, \varphi}(\Omega)=\lambda_{1, \varphi}^{*}(\Omega) \geq\left[\frac{(n-1) \cdot a-c^{+}}{2}\right]^{2},
$$

which, by [8, Lemma 1.1], implies the lower bounds for $\lambda_{1, p}(M), \lambda_{1, \varphi}(M)$ in Lemma 2.

REMARK 3. Clearly, when $p=2$ (or $\varphi=$ const.), the nonlinear $p$-Laplacian (or the weighted Laplacian) degenerate into the Laplacian. Correspondingly, $\lambda_{1, p}(M)=\lambda_{1}(M)$ (or $\lambda_{1, \varphi}(M)=\lambda_{1}(M), c^{+}=0$ ), and moreover, $\lambda_{1}(M) \geq \frac{(n-1)^{2} a^{2}}{4}>0$, which is exactly Mckean's lower bound shown in [17]. 


\section{Eigenvalue estimates for submanifolds with locally bounded mean curvature in Hadamard manifolds}

Let $\phi: M \rightarrow Q$ be an isometric immersion with $M, Q$ complete Riemannian manifolds, $\operatorname{dim}(M)=n, n \geq 2$. Consider a smooth function $g: Q \rightarrow \mathbb{R}$ and the composition $f=g \circ \phi: M \rightarrow \mathbb{R}$. As before, let $\Delta$ be the Laplace operator on $M$. However, because of the isometric immersion, for convenience, in this section, we can use $\operatorname{grad}(\cdot)$ to denote the gradient of a given function on $M$ or its isometric image $\phi(M) \subseteq Q$. Identify $X$ with $d \phi(X)$, and then we can obtain that at $q \in M$,

$$
\langle\operatorname{grad} f, X\rangle=d f(X)=d g(X)=\langle\operatorname{grad} g, X\rangle
$$

for every $X \in T_{q} M$. Therefore, it follows that

$$
\operatorname{grad} g=\operatorname{grad} f+(\operatorname{grad} g)^{\perp},
$$

with $(\operatorname{grad} g)^{\perp}$ perpendicular to $T_{q} M$. For $X, Y \in T_{q} M$, let $\alpha(q)(X, Y)$ and Hess $f(q)(X, Y)$ be the second fundamental form of the immersion $\phi$ and the Hessian of $f$ at $q \in M$, respectively. By the Gauss equation, we have

$$
\text { Hess } f(q)(X, Y)=\operatorname{Hess} g(\phi(q))(X, Y)+\langle\operatorname{grad} g, \alpha(X, Y)\rangle_{\phi(q)} .
$$

Taking the trace in (11) w.r.t. an orthonormal basis $\left\{e_{1}, e_{2} \ldots e_{n}\right\}$ of $T_{q} M$, we can get

$$
\begin{aligned}
\Delta f(q)=\sum_{i=1}^{n} \operatorname{Hess} f(q)\left(e_{i}, e_{i}\right)= & \sum_{i=1}^{n} \operatorname{Hess} g(\phi(q))\left(e_{i}, e_{i}\right) \\
& +\left\langle\operatorname{grad} g, \sum_{i=1}^{n} \alpha\left(e_{i}, e_{i}\right)\right\rangle .
\end{aligned}
$$

See, e.g., $[6,8]$ for more generalized versions of the formulas (11) and (12) above.

We need the following notion.

Definition 2. An isometric immersion $\phi: M \rightarrow Q$ has locally bounded mean curvature $H$ if for any $q \in Q$ and $r>0$, the number $h(q, r):=$ $\sup \left\{\|H(x)\| ; x \in \phi(M) \cap B_{Q}(q, r)\right\}$ is finite, where, as before, $B_{Q}(q, r)$ denotes the geodesic ball, with center $q$ and radius $r$, on $Q$.

By using Lemma 1, Theorem 1 and the locally bounded mean curvature assumption, we can prove the following. 
THEOREM 2. Let $\phi: M \rightarrow Q$ be an isometric immersion with locally bounded mean curvature and let $\Omega$ be any connected component of $\phi^{-1}\left(\overline{B_{Q}(q, r)}\right)$, where $q \in Q \backslash \phi(M), r>0$ and $\operatorname{dim}(M)=n, n \geq 2$. Let $\kappa(q, r)=\sup \left\{K_{Q}(x) \mid\right.$ $\left.x \in B_{Q}(q, r)\right\}$, where $K_{Q}(x)$ is the sectional curvature at $x$. Denote by $\operatorname{inj}(q)$ the injectivity radius of $Q$ at the point $q$. Assume that $\varphi$ is a real-valued smooth function on $M$ with $\|\operatorname{grad} \varphi\| \leq c^{+}$, where $c^{+}$is the supremum of the norm of the gradient of $\varphi$. Choosing $r$ properly, we have the following estimates:

(1) If $\kappa(q, \operatorname{inj}(q))=k^{2}<\infty, k>0$, choose

$$
r<\min \left\{\operatorname{inj}(q), \frac{\pi}{2 k}, \cot ^{-1}\left[\frac{h(q, \operatorname{inj}(q))}{(n-1) k}\right] / k\right\} .
$$

Then we have

$$
\lambda_{1, \varphi}^{*}(\Omega) \geq\left[\frac{(n-1) k \cot (k r)-h(q, r)-c^{+}}{2}\right]^{2}
$$

provided $c^{+}<(n-1) k \cot (k r)-h(q, r)$. If furthermore the boundary $\partial \Omega$ is piecewise smooth, then we have

$$
\lambda_{1, p}(\Omega) \geq\left[\frac{(n-1) k \cot (k r)-h(q, r)}{p}\right]^{p} .
$$

(2) If $\lim _{\ell \rightarrow \infty} \kappa(q, \ell)=\infty$, let

$$
r(s):=\min \left\{\frac{\pi}{2 \sqrt{\kappa(q, s)}}, \cot ^{-1}\left[\frac{h(q, s)}{(n-1) \sqrt{\kappa(q, s)}}\right] / \sqrt{\kappa(q, s)}\right\}, \quad s>0 .
$$

Choose $r=\max _{s>0} r(s)$. We have

$$
\lambda_{1, \varphi}^{*}(\Omega) \geq\left[\frac{(n-1) \sqrt{\kappa(q, s)} \cot (\sqrt{\kappa(q, s)} r)-h(q, r)-c^{+}}{2}\right]^{2}
$$

provided $c^{+}<(n-1) \sqrt{\kappa(q, s)} \cot (\sqrt{\kappa(q, s)} r)-h(q, r)$. If furthermore the boundary $\partial \Omega$ is piecewise smooth, then we have

$$
\lambda_{1, p}(\Omega) \geq\left[\frac{(n-1) \sqrt{\kappa(q, s)} \cot (\sqrt{\kappa(q, s)} r)-h(q, r)}{p}\right]^{p} .
$$

(3) If $\kappa(q, \operatorname{inj}(q))=0$, choose $r<\min \left\{\operatorname{inj}(q), \frac{n}{h(q, \operatorname{inj}(q))}\right\}$. Assume that $\frac{n}{h(q, \operatorname{inj}(q))}=\infty$ if $h(q, \operatorname{inj}(q))=0$. Then we have

$$
\lambda_{1, \varphi}^{*}(\Omega) \geq\left[\frac{\frac{n}{r}-h(q, r)-c^{+}}{2}\right]^{2}
$$


provided $c^{+}<\frac{n}{r}-h(q, r)$. If furthermore $\Omega$ is bounded and its boundary $\partial \Omega$ is piecewise smooth, then we have

$$
\lambda_{1, p}(\Omega) \geq\left[\frac{\frac{n}{r}-h(q, r)}{p}\right]^{p} .
$$

(4) If $\kappa(q, \operatorname{inj}(q))=-k^{2}<\infty, k>0$, and $h(q, \operatorname{inj}(q))<(n-1) k$, choose $r<\operatorname{inj}(q)$. Then

$$
\lambda_{1, \varphi}^{*}(\Omega) \geq\left[\frac{(n-1) k-h(q, r)-c^{+}}{2}\right]^{2}
$$

provided $c^{+}<(n-1) k-h(q, r)$. If furthermore $\Omega$ is bounded and its boundary $\partial \Omega$ is piecewise smooth, then we have

$$
\lambda_{1, p}(\Omega) \geq\left[\frac{(n-1) k-h(q, r)}{p}\right]^{p} .
$$

(5) If $\kappa(q, \operatorname{inj}(q))=-k^{2}<\infty, k>0$, and $h(q, \operatorname{inj}(q)) \geq(n-1) k$, choose

$$
r<\min \left\{\operatorname{inj}(q), \operatorname{coth}^{-1}\left[\frac{h(q, \operatorname{inj}(q))}{(n-1) k}\right] / k\right\} .
$$

Then we have

$$
\lambda_{1, \varphi}^{*}(\Omega) \geq\left[\frac{(n-1) k \operatorname{coth}(k r)-h(q, r)-c^{+}}{2}\right]^{2}
$$

provided $c^{+}<(n-1) k \operatorname{coth}(k r)-h(q, r)$. If furthermore the boundary $\partial \Omega$ is piecewise smooth, then we have

$$
\lambda_{1, p}(\Omega) \geq\left[\frac{(n-1) k \operatorname{coth}(k r)-h(q, r)}{p}\right]^{p} .
$$

In (2), since $r(s)>0$ for small $s, r>0$. In (3)-(5), because of the non-positivity assumption on $\kappa(q, \operatorname{inj}(q))$, the radius $r$ is not necessary to be finite, which implies that the connected component $\Omega$ of $\phi^{-1}\left(\overline{B_{Q}(q, r)}\right)$ may be unbounded as $r \rightarrow \infty$. Besides, in (4), one can have a slight better estimate as follows

$$
\lambda_{1, \varphi}^{*}(\Omega) \geq\left[\frac{(n-1) k+\frac{1}{r}-h(q, r)-c^{+}}{2}\right]^{2}
$$

provided $c^{+}<(n-1) k+\frac{1}{r}-h(q, r)$, by choosing $X=\operatorname{grad}\left(\rho^{2} \circ \phi\right)$ in the proof below. 
Proof. Similar to the proof of [1, Theorem 4.3]. Define two functions as follows

$$
f_{i}=\rho^{i} \circ \phi: M \rightarrow \mathbb{R}, \quad i=1,2,
$$

where $\rho(x)=\operatorname{dist}_{Q}(q, x)$ is the distance function on $Q$. Clearly, $f_{1}, f_{2}$ are smooth functions on $\phi^{-1}\left(B_{Q}(q, \operatorname{inj}(q))\right)$. Let $\Omega$ be a connected component of $\phi^{-1}\left(\overline{B_{Q}(q, r)}\right) \subseteq \phi^{-1}\left(B_{Q}(q, \operatorname{inj}(q))\right)$, and let $X_{i}=\operatorname{grad} f_{i}, i=1,2$, on $\Omega$. By (12), we have

$$
\operatorname{div} X_{i}(x)=\Delta f_{i}(x)=\sum_{j=1}^{n-1} \operatorname{Hess} \rho^{i}(\phi(x))\left(e_{j}, e_{j}\right)+\left\langle\operatorname{grad} \rho^{i}, H\right\rangle_{\phi(x)},
$$

with $\left\{e_{1}, e_{2}, \ldots, e_{n}\right\}$ an orthonormal basis of $T_{x} M$, where $e_{n}=\operatorname{grad} \rho(x)$. Applying Theorem 1 directly, one can obtain

- if $\kappa(q, \operatorname{inj}(q))=k^{2}<\infty, k>0$, then $\operatorname{div} X_{1} \geq(n-1) k \cot (k r)-h(q, r)$ $>0$;

- if $\kappa(q, \operatorname{inj}(q))=0$, then $\operatorname{div} X_{2} \geq 2 n-2 r h(q, r)>0$;

- if $\kappa(q, \operatorname{inj}(q))=-k^{2}<\infty, k>0$, then $\operatorname{div} X_{1} \geq(n-1) k \operatorname{coth}(k r)-$ $h(q, r)>0$.

Together with the fact that $\left\|X_{1}\right\|=1$ and $\left\|X_{2}\right\|=2 r$, estimates in Theorem 2 can be obtained by applying Lemma 1 directly.

REMARK 4. Clearly, when $\varphi=$ const. (or $p=2, \Omega$ is bounded), our estimates here are exactly those in [1, Theorem 4.3].

Applying directly Theorem 2, we can obtain

Corollary 3. Let $\phi: M \rightarrow \mathbb{R}^{m}$ be an isometric minimal immersion of an $n$-dimensional $(n \geq 2)$ complete submanifold. Assume that $\phi(M) \subset B_{\mathbb{R}^{m}}(o, r)$, then $\lambda_{1, p}(M) \geq\left(\frac{n}{p r}\right)^{p}$.

Using a similar proof to that of [1, Corollary 4.4] and applying directly Theorem 2, [11, Proposition 10.1], [18, Theorem A.3], we can get the following.

Corollary 4. Let $\phi: M \rightarrow Q$ be an isometric immersion with bounded mean curvature $\|H\| \leq \alpha<(n-1) a$, where $M$ is an $n$-dimensional complete noncompact Riemannian manifold and $Q$ is an m-dimensional complete simply connected Riemannian manifold with sectional curvature $K_{Q}$ satisfying $K_{Q} \leq-a^{2}<$ 0 for some constant $a>0$. Assume that $\varphi$ is a real-valued smooth function on $M$ with $\|\operatorname{grad} \varphi\| \leq c^{+}$, where $c^{+}$is the supremum of the norm of the gradient of $\varphi$. Then we have the following estimates

$$
\lambda_{1, \varphi}(M) \geq\left[\frac{(n-1) a-\alpha-c^{+}}{2}\right]^{2}>0 \quad\left(\text { provided } c^{+}<(n-1) a-\alpha\right)
$$


and

$$
\lambda_{1, p}(M) \geq\left[\frac{(n-1) a-\alpha}{p}\right]^{p}>0 .
$$

In particular, there exist entire Green's functions on $M$. If furthermore $M$ is minimal, then $M$ is non-parabolic.

REMARK 5. Corollary 4 gives a positive answer to Questions 1 and 2 proposed in Section 1, i.e., finding conditions such that $\lambda_{1, \varphi}(M)>0, \lambda_{1, p}(M)>$ 0 for a complete noncompact manifold $M$, and also shows interesting geometric conclusions, i.e., the existence of Green's functions and the non-parabolic property. Besides, if $Q=\mathbb{H}^{m}(-1)$ which implies $a=1$, then our lower bounds here are exactly those in [8, Theorems 1.3 and 1.7].

\section{Eigenvalue estimates for submanifolds with locally bounded mean curvature in product manifolds $N \times \mathbb{R}$}

Let $\phi: M \rightarrow N \times \mathbb{R}$ be an isometric immersion from an $n$-dimensional complete Riemannian manifold to the product space $N \times \mathbb{R}$ with $N$ an $m$-dimensional complete Riemannian manifold. Since $\phi$ is an isometric immersion, we have formulas (11), (12) with $Q=N \times \mathbb{R}$. Besides, for convenience, we can use $\operatorname{grad}(\cdot)$ to denote the gradient of a given function on $M$ or its isometric image $\phi(M) \subseteq N \times \mathbb{R}$. In this section, we would like to estimate from below the first fundamental tone $\lambda_{1, \varphi}^{*}(\Omega)$ of $\Omega$ (with $\Omega \subseteq M$ ) and the first eigenvalue $\lambda_{1, p}(\Omega)$ of the $p$-Laplacian on $\Omega$ (with $\Omega \subset M$ a domain with compact closure and piecewise smooth boundary). However, before that, we need the following notion, which is stronger than the one in Definition 2.

Definition 3 ([3]). An isometric immersion $\phi: M \rightarrow N \times \mathbb{R}$ has locally bounded mean curvature $H$ if for any $q \in N$ and $r>0$, the number $h(q, r):=$ $\sup \left\{\|H(x)\| ; x \in \phi(M) \cap\left(B_{N}(q, r) \times \mathbb{R}\right)\right\}$ is finite, where $B_{N}(q, r)$ denotes the geodesic ball, with center $q$ and radius $r$, on $N$.

We also need the following conclusion, which is an extension of [2, Theorem 1.7].

Lemma 3. Let $\mathscr{W}^{1,1}(M)$ be the Sobolev space of all vector fields $X \in$ $L_{\text {loc }}^{1}(M)$ possessing weak divergence ${ }^{2} \operatorname{div} X$ on a Riemannian manifold $M$.

\footnotetext{
${ }^{2}$ For a Riemannian manifold $M$, a function $g \in L_{l o c}^{1}(M)$ is a weak divergence of $X$ if $\int_{M} g \psi=$ $-\int_{M}\langle\operatorname{grad} \psi, X\rangle, \forall \psi \in C_{0}^{\infty}(M)$. There exists at most one $g \in L_{l o c}^{1}(M)$ for a given vector field $X \in L_{\text {loc }}^{1}(M)$ and we can write $g=\operatorname{div} X$. Clearly, for a $C^{1}$ vector field $X$, its classical divergence coincides with the weak divergence $\operatorname{div} X$.
} 
Assume that $\varphi$ is a real-valued smooth function on $M$ with $\|\operatorname{grad} \varphi\| \leq c^{+}$, where $c^{+}$is the supremum of the norm of the gradient of $\varphi$. Then the weighted fundamental tone $\lambda_{1, \varphi}^{*}(M)$ of $M$ satisfies

$$
\lambda_{1, \varphi}^{*}(M) \geq \sup _{\mathscr{W}^{1,1}(M)}\left\{\inf _{M}\left(\operatorname{div} X-\|X\|^{2}-c^{+}\|X\|\right)\right\} .
$$

If furthermore $M$ is complete, then the first eigenvalue $\lambda_{1, p}(M)$ of the p-Laplacian satisfies

$$
\lambda_{1, p}(M) \geq \sup _{\mathscr{W}^{1,1}(M)}\left\{\inf _{M}\left[\operatorname{div} X-(p-1)\|X\|^{p /(p-1)}\right]\right\} .
$$

Proof. Let $X \in L_{\text {loc }}^{1}(M)$ and $f \in C_{0}^{\infty}(M)$. Clearly, we have $\int_{M} \operatorname{div}\left(f^{2} X e^{-\varphi}\right)=0$ and $\int_{M} \operatorname{div}\left(|f|^{p} X\right)=0$. By a direct computation, it follows that

$$
\begin{aligned}
0 & =\int_{M} \operatorname{div}\left(f^{2} X e^{-\varphi}\right) \\
& =\int_{M} f^{2} \operatorname{div} X \cdot e^{-\varphi}+\int_{M}\left\langle\operatorname{grad} f^{2}, X\right\rangle e^{-\varphi}-\int_{M} f^{2}\langle\operatorname{grad} \varphi, X\rangle e^{-\varphi} \\
& \geq \int_{M} f^{2} \operatorname{div} X \cdot e^{-\varphi}-2 \int_{M}|f| \cdot\|X\| \cdot\|\operatorname{grad} f\| e^{-\varphi}-c^{+} \int_{M}\|X\| f^{2} e^{-\varphi} \\
& \geq \int_{M} f^{2} \operatorname{div} X \cdot e^{-\varphi}-\int_{M}\left[f^{2} \cdot\|X\|^{2}+\|\operatorname{grad} f\|^{2}\right] e^{-\varphi}-c^{+} \int_{M}\|X\| f^{2} e^{-\varphi} \\
& =\int_{M}\left(\operatorname{div} X-\|X\|^{2}-c^{+}\|X\|\right) f^{2} e^{-\varphi}-\int_{M}\|\operatorname{grad} f\|^{2} e^{-\varphi} \\
& \geq \inf _{M}\left(\operatorname{div} X-\|X\|^{2}-c^{+}\|X\|\right) \int_{M} f^{2} e^{-\varphi}-\int_{M}\|\operatorname{grad} f\|^{2} e^{-\varphi}
\end{aligned}
$$

which implies

$$
\frac{\int_{M}\|\operatorname{grad} f\|^{2} e^{-\varphi}}{\int_{M} f^{2} e^{-\varphi}} \geq \inf _{M}\left(\operatorname{div} X-\|X\|^{2}-c^{+}\|X\|\right) .
$$

Then, by taking supremum to both sides of the above inequality over $\mathscr{W}^{1,1}(M)$, we have

$$
\frac{\int_{M}\|\operatorname{grad} f\|^{2} e^{-\varphi}}{\int_{M} f^{2} e^{-\varphi}} \geq \sup _{\mathscr{W}^{1,1}(M)}\left\{\inf _{M}\left(\operatorname{div} X-\|X\|^{2}-c^{+}\|X\|\right)\right\},
$$

which implies (13). On the other hand, since $\int_{M} \operatorname{div}\left(|f|^{p} X\right)=0$, by a direct calculation, one can obtain 


$$
\begin{aligned}
0=\int_{M} \operatorname{div}\left(|f|^{p} X\right) & =\int_{M}\left\langle\operatorname{grad}\left(|f|^{p}\right), X\right\rangle+\int_{M}|f|^{p} \operatorname{div} X \\
& \geq-\int_{M} p|f|^{p-1}\|\operatorname{grad} f\| \cdot\|X\|+\int_{M}|f|^{p} \operatorname{div} X \\
& \geq-\int_{M} p\left[\frac{\left(|f|^{p-1}\|X\|\right)^{p /(p-1)}}{\frac{p}{p-1}}+\frac{\|\operatorname{grad} f\|^{p}}{p}\right]+\int_{M}|f|^{p} \operatorname{div} X \\
& =\int_{M}\left[\operatorname{div} X-(p-1)\|X\|^{p /(p-1)}\right]|f|^{p}-\int_{M}\|\operatorname{grad} f\|^{p} \\
& \geq \inf _{M}\left[\operatorname{div} X-(p-1)\|X\|^{p /(p-1)}\right] \int_{M}|f|^{p}-\int_{M}\|\operatorname{grad} f\|^{p},
\end{aligned}
$$

where the second inequality holds by applying Young's inequality. Therefore, we have

$$
\frac{\int_{M}\|\operatorname{grad} f\|^{p}}{\int_{M}|f|^{p}} \geq \inf _{M}\left[\operatorname{div} X-(p-1)\|X\|^{p /(p-1)}\right],
$$

and then, by taking supremum to both sides of the above inequality over $\mathscr{W}^{1,1}(M)$, we have

$$
\frac{\int_{M}\|\operatorname{grad} f\|^{p}}{\int_{M}|f|^{p}} \geq \sup _{\mathscr{W}^{1,1}(M)}\left\{\inf _{M}\left[\operatorname{div} X-(p-1)\|X\|^{p /(p-1)}\right]\right\}
$$

which implies (14). This completes the proof of Lemma 3.

REMARK 6. (1) Using an almost same method, we can get

$$
\lambda_{1, \varphi}^{*}(M) \geq \sup _{\mathscr{W}^{1,1}(M)}\left\{\inf _{M \backslash F}\left(\operatorname{div} X-\|X\|^{2}-c^{+}\|X\|\right)\right\}
$$

and

$$
\lambda_{1, p}(M) \geq \sup _{\mathscr{W}^{1,1}(M)}\left\{\inf _{M \backslash F}\left[\operatorname{div} X-(p-1)\|X\|^{p /(p-1)}\right]\right\},
$$

where $F$ has zero Riemannian volume.

(2) If $M$ is compact, then, by taking infimum to the LHS of (15) over the space $\left\{f \mid f \in W_{0}^{1, p}(\Omega), f \neq 0\right\}$, one can get (14) directly. If $M$ is noncompact, one can choose an exhaustion $\left\{\Omega_{i}\right\}_{i=1,2,3, \ldots}$ with $\Omega_{i} \subset \Omega_{j}, i<j$, then as the compactness situation, one can obtain

$$
\lambda_{1, p}\left(\Omega_{i}\right) \geq \sup _{\mathscr{W}^{1,1}\left(\Omega_{i}\right)}\left\{\inf _{\Omega_{i}}\left[\operatorname{div} X-(p-1)\|X\|^{p /(p-1)}\right]\right\},
$$


which, by applying domain monotonicity of the first eigenvalue of the $p$-Laplacian with vanishing Dirichlet data and taking limits to both sides of the above inequality as $i \rightarrow \infty$, implies (14).

For clarifying argument below better, we need to define functions $S_{k}(t)$ and $C_{k}(t)$ as follows.

$$
S_{k}(t)= \begin{cases}\sin (\sqrt{k} \cdot t) / \sqrt{k}, & \text { if } k>0, \\ t, & \text { if } k=0, \\ \sinh (\sqrt{-k} \cdot t) / \sqrt{-k}, & \text { if } k<0\end{cases}
$$

and

$$
C_{k}(t)=S_{k}^{\prime}(t)
$$

We can prove the following.

THEOREM 3. Let $\phi: M \rightarrow N \times \mathbb{R}$ be an $n$-dimensional $(n \geq 3)$ complete minimal isometric immersed submanifold, where the m-dimensional Riemannian manifold $N$ has radial sectional curvature $K_{\gamma(t)}\left(\gamma^{\prime}(t), \vec{v}\right) \leq k, \vec{v} \in T_{\gamma(t)} N,\|\vec{v}\|=1$, $\vec{v} \perp \frac{\partial}{\partial t}$, along the minimizing geodesic $\gamma(t)$ issuing from a point $q \in N$. Let $\Omega$ be any connected component of $\phi^{-1}\left(B_{N}(q, r) \times \mathbb{R}\right)$, where $r<\min \left\{\operatorname{inj}_{N}(q), \frac{\pi}{2 \sqrt{k}}\right\}$ $(\pi / 2 \sqrt{k}=\infty$ if $k \leq 0)$, and $\operatorname{inj}_{N}(q)$ denotes the injectivity radius of $N$ at the point $q$. Assume that $\varphi$ is a real-valued smooth function on $M$ with $\|\operatorname{grad} \varphi\| \leq$ $c^{+}$, where $c^{+}$is the supremum of the norm of the gradient of $\varphi$. Suppose in addition that

- if $|h(q, r)|<F^{2}<\infty$, then $r \leq\left(\frac{C_{k}}{S_{k}}\right)^{-1} \cdot \frac{F^{2}}{(n-2)}$ or

- if $\lim _{r \rightarrow \infty} h\left(q, r_{0}\right)=\infty$, then $r \leq\left(\frac{C_{k}}{S_{k}}\right)^{-1} \cdot \frac{h\left(q, r_{0}\right)}{(n-2)}$, where $r_{0}$ is chosen such that $(n-2) \frac{C_{k}\left(r_{0}\right)}{S_{k}\left(r_{0}\right)}-h\left(q, r_{0}\right)=0$.

Then we have

$$
\lambda_{1, \varphi}^{*}(\Omega) \geq\left[\frac{(n-2) \frac{C_{k}(r)}{S_{k}(r)}-h(q, r)-c^{+}}{2}\right]^{2}
$$

provided $c^{+}<(n-2) \frac{C_{k}(r)}{S_{k}(r)}-h(q, r)$, and

$$
\lambda_{1, p}(\Omega) \geq\left[\frac{(n-2) \frac{C_{k}(r)}{S_{k}(r)}-h(q, r)}{p}\right]^{p} .
$$

Proof. Define a function $\tilde{\rho}: N \times \mathbb{R} \rightarrow \mathbb{R}$ by $\tilde{\rho}(x, t)=\rho_{N}(x)$, where $\rho_{N}(x)=\operatorname{dist}_{N}(q, x)$ is the distance function in $N$ to the point $x_{0}$. Let $\Omega \subset$ $\phi^{-1}\left(B_{N}(q, r) \times \mathbb{R}\right), f=\tilde{\rho} \circ \phi$ and $X=\operatorname{grad} f$. Properly choose $r$ such that 
$\inf _{\Omega} \operatorname{div} X>0$. As before, denote by $\Delta$ the Laplacian on $M$. Clearly, $\Delta f=\operatorname{div} X$. By Lemma 1 , we have

$$
\lambda_{1, \varphi}^{*}(\Omega) \geq\left(\frac{\inf \operatorname{div} X}{2 \sup \|X\|}-\frac{c^{+}}{2}\right)^{2}
$$

and

$$
\lambda_{1, p}(\Omega) \geq\left(\frac{\inf \operatorname{div} X}{p \sup \|X\|}\right)^{p} .
$$

Consider the orthonormal basis $\left\{\operatorname{grad} \rho_{N}, \frac{\partial}{\partial \theta_{1}}, \ldots, \frac{\partial}{\partial \theta_{m-1}}, \frac{\partial}{\partial s}\right\}$ for the tangent space $T_{(q, s)}(N \times \mathbb{R})$ with $\phi(w)=(q, s)$, where $\left\{\operatorname{grad} \rho_{N}, \frac{\partial}{\partial \theta_{1}}, \ldots, \frac{\partial}{\partial \theta_{m-1}}\right\}$ is the polar coordinates for $T_{q} N$. Denote by $\left\{e_{1}, e_{2}, \ldots, e_{n}\right\}$ an orthonormal basis for $T_{w} \Omega$. Then one can decompose $e_{i}$ as follows

$$
e_{i}=a_{i} \cdot \operatorname{grad} \rho_{N}+b_{i} \cdot \frac{\partial}{\partial s}+\sum_{j=1}^{m-1} c_{i}^{j} \cdot \frac{\partial}{\partial \theta_{j}}, \quad i=1,2, \ldots, n,
$$

where $a_{i}, b_{i}, c_{i}^{j}$ are constants satisfying

$$
a_{i}^{2}+b_{i}^{2}+\sum_{j=1}^{m-1}\left(c_{i}^{j}\right)^{2}=1 .
$$

By applying (12) with $Q=N \times \mathbb{R}$ to the function $f$, it follows that

$$
\Delta f=\left[\sum_{i=1}^{n} \operatorname{Hess}_{N \times \mathbb{R}} \tilde{\rho}\left(e_{i}, e_{i}\right)+\left\langle\operatorname{grad}_{N \times \mathbb{R}} \tilde{\rho}, H\right\rangle\right]_{\phi(w)},
$$

where $H=\sum_{i=1}^{n} \alpha\left(e_{i}, e_{i}\right)$ is the mean curvature vector of $\phi(M)$ at the point $\phi(w)$ and the orthonormal basis $\left\{e_{1}, e_{2}, \ldots, e_{n}\right\}$ of $T_{w} M$ identified with $\left\{\phi_{*}\left(e_{1}\right)\right.$, $\left.\phi_{*}\left(e_{2}\right), \ldots, \phi_{*}\left(e_{n}\right)\right\}$. By Theorem 1 and $(20)$, we have

$$
\begin{aligned}
\sum_{i=1}^{n} \operatorname{Hess}_{N \times \mathbb{R}} \tilde{\rho}\left(e_{i}, e_{i}\right) & =\sum_{i=1}^{n} \operatorname{Hess}_{N} \rho_{N}\left(e_{i}, e_{i}\right) \\
& =\sum_{i=1}^{n} \sum_{j=1}^{m-1}\left(c_{i}^{j}\right)^{2} \operatorname{Hess}_{N} \rho_{N}\left(\frac{\partial}{\partial \theta_{i}}, \frac{\partial}{\partial \theta_{j}}\right) \\
& \geq \sum_{i=1}^{n}\left(1-a_{i}^{2}-b_{i}^{2}\right) \frac{C_{k}(r)}{S_{k}(r)}
\end{aligned}
$$

and 


$$
\begin{aligned}
\left\langle\operatorname{grad}_{N \times \mathbb{R}} \tilde{\rho}, H\right\rangle=\left\langle\operatorname{grad}_{N} \rho_{N}, H\right\rangle & =\left\langle\left(\operatorname{grad}_{N} \rho_{N}\right)^{\perp}, H\right\rangle \\
& \leq\left\|\left(\operatorname{grad}_{N} \rho_{N}\right)^{\perp}\right\| \cdot\|H\|=\|H\| \sqrt{1-\sum_{i=1}^{n} a_{i}^{2}} \\
& \leq h\left(x_{0}, r\right) \sqrt{1-\sum_{i=1}^{n} a_{i}^{2}}
\end{aligned}
$$

Substituting the above two inequalities into (21), together with the fact $1-\sum_{i=1}^{n} a_{i}^{2} \geq 0$ and $1-\sum_{i=1}^{n} b_{i}^{2} \geq 0$, yields

$$
\Delta f \geq(n-2) \frac{C_{k}(r)}{S_{k}(r)}-h(q, r)>0 .
$$

If $\left|h\left(x_{0}, r\right)\right|<F^{2}<\infty$, then we can choose

$$
r \leq\left\{\operatorname{inj}_{N}(q), \frac{\pi}{2 \sqrt{k}},\left(\frac{C_{k}}{S_{k}}\right)^{-1} \cdot \frac{F^{2}}{(n-2)}\right\}
$$

If $\lim _{r \rightarrow \infty} h\left(q, r_{0}\right)=\infty$, there exists $r_{0}$ such that $(n-2) \frac{C_{k}\left(r_{0}\right)}{S_{k}\left(r_{0}\right)}-h\left(q, r_{0}\right)=0$ since $h(q, r)$ is a continuous nondecreasing function in $r$. Then in this situation, we can choose

$$
r \leq\left\{\operatorname{inj}_{N}(q), \frac{\pi}{2 \sqrt{k}},\left(\frac{C_{k}}{S_{k}}\right)^{-1} \cdot \frac{h\left(q, r_{0}\right)}{(n-2)}\right\} .
$$

Putting (22) with $\operatorname{div} X=\Delta f$ into (18) and (19), our estimates for $\lambda_{1, \varphi}^{*}(\Omega)$ and $\lambda_{1, p}(\Omega)$ can be obtained.

REMARK 7. If $\Omega$ is bounded and has the piecewise smooth boundary, then putting (22) with $\operatorname{div} X=\Delta f$ into (19), the estimate (14) follows. If $\Omega$ is unbounded, one can choose an exhaustion $\left\{\Omega_{i}\right\}_{i=1,2,3, \ldots}$ with $\Omega_{i} \subset \Omega_{j} \subset \Omega$, $i<j$, and putting (22) into (19) for the bounded domain $\Omega_{i}$, we have

$$
\lambda_{1, p}\left(\Omega_{i}\right) \geq\left[\frac{(n-2) \frac{C_{k}(r)}{S_{k}(r)}-h(q, r)}{p}\right]^{p},
$$

which implies the estimate (14) by applying domain monotonicity of the first eigenvalue of the $p$-Laplacian with vanishing Dirichlet data and taking limits to both sides of the above inequality as $i \rightarrow \infty$. Besides, clearly, when $\varphi=$ const. or $p=2$, our estimates here are exactly the one in $[2$, Theorem $1.6]$. 


\section{Eigenvalue estimates for submanifolds with bounded $\varphi$-mean curvature} in the hyperbolic space

For an $n$-dimensional $(n \geq 2)$ submanifold $M$ of the weighted manifold

$$
\left(\mathbb{H}^{m}(-1), e^{-\varphi} d v\right),
$$

its $\varphi$-mean curvature vector field $H_{\varphi}$ is given by

$$
H_{\varphi}:=H+(\bar{\nabla} \varphi)^{\perp}
$$

where $\perp$ denotes the projection onto the normal bundle of $M, \bar{\nabla}$ is the gradient operator on the hyperbolic $m$-space $\mathbb{H}^{m}(-1)$, and, as before, $H$ is the mean curvature vector of $M$. We call $M$ is $\varphi$-minimal if $H_{\varphi}$ vanishes everywhere. See, e.g., $[12,19]$ for the notion of $\varphi$-mean curvature and some interesting applications.

REMARK 8. Clearly, if $\varphi=$ const., then $H_{\varphi}=H$, and in this situation, "minimal" is equivalent to " $\varphi$-minimal". However, in general case, they are different.

Now, by applying the $\varphi$-minimal assumption and [8, Theorem 1.3], we can prove the following result.

THEOREM 4. Let $M$ be an $n$-dimensional $(n \geq 2)$ complete noncompact $\varphi$-minimal submanifold of the weighted manifold $\left(\mathbb{H}^{m}(-1), e^{-\varphi} d v\right)$. If $\sup _{M}\|\bar{\nabla} \varphi\|<n-1$, then

$$
\lambda_{1, p}(M) \geq\left(\frac{n-1-\sup _{M}\|\bar{\nabla} \varphi\|}{p}\right)^{p}>0 .
$$

Proof. By a direct calculation, we have

$$
\sup _{M}\|H\| \leq \sup _{M} \sqrt{\|H\|^{2}+\left\|(\bar{\nabla} \varphi)^{\top}\right\|^{2}}=\sup _{M}\left\|H+(\bar{\nabla} \varphi)^{\top}\right\|=\sup _{M}\left\|H_{\varphi}-\bar{\nabla} \varphi\right\|,
$$

where $T$ denotes the projection onto the tangent bundle of $M$. Therefore, if $M$ is $\varphi$-minimal and $\sup _{M}\|\bar{\nabla} \varphi\|<n-1$, then $\sup _{M}\|H\|<n-1$. By applying [8, Theorem 1.3] directly, we have

$$
\lambda_{1, p}(M) \geq\left(\frac{n-1-\sup _{M}\|H\|}{p}\right)^{p}>0 .
$$

This implies 


$$
\begin{aligned}
\lambda_{1, p}(M) & \geq\left[\frac{n-1-\sup _{M} \sqrt{\|H\|^{2}+\left\|(\bar{\nabla} \varphi)^{\top}\right\|^{2}}}{p}\right]^{p} \\
& =\left(\frac{n-1-\sup _{M}\left\|H_{\varphi}-\bar{\nabla} \varphi\right\|}{p}\right)^{p} \\
& =\left(\frac{n-1-\sup _{M}\|\bar{\nabla} \varphi\|}{p}\right)^{p}>0
\end{aligned}
$$

provided $M$ is $\varphi$-minimal and $\sup _{M}\|\bar{\nabla} \varphi\|<n-1$.

REMARK 9. Clearly, when $\varphi=$ const., our estimate (23) becomes

$$
\lambda_{1, p}(M) \geq\left(\frac{n-1}{p}\right)^{p}>0,
$$

which is exactly (1.5) of [8]. When $\varphi=$ const. and $p=2$, our Theorem 4 degenerate into [6, Corollary 3$]$.

\section{Lower bounds for the first Dirichlet eigenvalues of the weighted \\ Laplacian and the $p$-Laplacian on geodesic balls}

For an $n$-dimensional $(n \geq 2)$ complete Riemannian manifold $M$ with sectional curvature bounded from above by some constant $k$, Cheng [5] proved $\lambda_{1}\left(B_{M}(q, r)\right) \geq \lambda_{1}\left(B_{\mathscr{M}(n, k)}(r)\right)$ with equality holds if and only if $B_{M}(q, r)$ is isometric to $B_{\mathscr{M}(n, k)}(r)$, where $B_{M}(q, r)$ is the geodesic ball, with center $q \in M$ and radius $r$, within the cut-locus of $q, B_{\mathscr{M}(n, k)}(r)$ is the geodesic ball of radius $r$ in the $n$-dimensional space form $\mathscr{M}(n, k)$ with constant sectional curvature $k$. By using the radial sectional curvature (whose upper bound is given by a continuous function of the Riemannian distance parameter) assumption and spherically symmetric manifolds as model spaces, Freitas, Mao and Salavessa [10, Theorem 4.4] improved Cheng's conclusion mentioned above a lot. The advantage of Freitas-Mao-Salavessa's theory has been shown intuitively by numerically calculating the first Dirichlet eigenvalue of the Laplacian on torus, elliptic paraboloid and saddle (see [10, Section 6]). Besides, the principle of doing numerical calculation for the first Dirichlet eigenvalue of the Laplacian on parameterized surfaces has been given in $[9,13]$.

It is well-known that the first Dirichlet eigenvalue $\lambda_{1}\left(B_{\mathbb{R}^{n}}(r)\right)$ of the Laplacian of a ball in $\mathbb{R}^{n}$ with radius $r$ is $\lambda_{1}\left(B_{\mathbb{R}^{n}}(r)\right)=\left(\frac{J_{n / 2-1}}{r}\right)^{2}$, where $J_{n / 2-1}$ is the first zero point of the $\left(\frac{n}{2}-1\right)$-st Bessel function. By Cheng's eigenvalue 
comparison [5] (or its generalization [10, Theorem 4.4]), for an $n$-dimensional $(n \geq 2)$ complete Riemannian manifold $M$ with non-positive sectional curvature, one has

$$
\lambda_{1}\left(B_{M}(q, r)\right) \geq\left(\frac{J_{n / 2-1}}{r}\right)^{2}
$$

where the geodesic ball $B_{M}(q, r)$ is within the cut-locus of $q \in M$. The equality in (24) holds if and only if $B_{M}(q, r)$ is isometric to $B_{\mathbb{R}^{n}}(r)$. bounds.

However, applying Lemma 1, we can prove the following sharper lower

THEOREM 5. Let $M$ be an $n$-dimensional $(n \geq 2)$ complete manifold and a point $q \in M$. Let $B_{M}(q, r)$ be a geodesic ball with center $q \in M$ and radius $r$, where $r<\operatorname{inj}(\mathrm{q})$ with $\operatorname{inj}(\mathrm{q})$ the injective radius of $q$. Let $\kappa(q, r)=$ $\sup \left\{K_{M}(x) \mid x \in B_{M}(q, r)\right\}$, where $K_{M}(x)$ are sectional curvatures of $M$ at $x$. Assume that $\varphi$ is a real-valued smooth function on $M$ with $\|\nabla \varphi\| \leq c^{+}$, where $c^{+}$is the supremum of the norm of the gradient of $\varphi$. Then for $k>0$, we have

$$
\begin{aligned}
& \lambda_{1, \varphi}\left(B_{M}(q, r)\right) \\
& \geq \begin{cases}\frac{1}{4} \cdot\left[(n-1) k \operatorname{coth}(k r)+\frac{1}{r}-c^{+}\right]^{2}, & \text { if } \kappa(q, r)=-k^{2}, \\
\left(\frac{n}{2 r}-\frac{c^{+}}{2}\right)^{2}, & \text { if } \kappa(q, r)=0 \text { and } \lambda_{1, \varphi}(M)>0, \\
{\left[\frac{(n-1) k r \cot (k r)+1}{2 r}-\frac{c^{+}}{2}\right]^{2},} & \text { if } \kappa(q, r)=k^{2} \text { and } r<\frac{\pi}{2 k}\end{cases}
\end{aligned}
$$

and

$$
\begin{aligned}
& \lambda_{1, p}\left(B_{M}(q, r)\right) \\
& \geq \begin{cases}\left(\frac{1}{p}\right)^{p} \cdot\left[(n-1) k \operatorname{coth}(k r)+\frac{1}{r}\right]^{p}, & \text { if } \kappa(q, r)=-k^{2}, \\
\left(\frac{n}{p r}\right)^{p}, & \text { if } \kappa(q, r)=0 \text { and } \lambda_{1, p}(M)>0, \\
{\left[\frac{(n-1) k r \cot (k r)+1}{p r}\right]^{p},} & \text { if } \kappa(q, r)=k^{2} \text { and } r<\frac{\pi}{2 k},\end{cases}
\end{aligned}
$$

where $c^{+}$satisfies

$$
c^{+}< \begin{cases}(n-1) k \operatorname{coth}(k r)+\frac{1}{r}, & \text { if } \kappa(q, r)=-k^{2}, \\ \frac{n}{r}, & \text { if } \kappa(q, r)=0 \text { and } \lambda_{1, \varphi}(M)>0, \\ \frac{(n-1) k r \cot (k r)+1}{r}, & \text { if } \kappa(q, r)=k^{2} \text { and } r<\frac{\pi}{2 k} .\end{cases}
$$


Proof. As before, let $\nabla$ and $\Delta$ be the gradient and the Laplace operators on $M$ respectively. Choose $X=\nabla \rho^{2}$ with $\rho(x)=\operatorname{dist}_{M}(q, x)$. Then $\|X\|=$ $2 \rho\|\nabla \rho\|=2 \rho$. By (10), we have

$$
\begin{aligned}
& \operatorname{div} X=\Delta \rho^{2} \geq 2(n-1) \rho \cdot \frac{1}{\rho}+2=2 n, \quad \text { if } \kappa(q, r)=0, \\
& \operatorname{div} X=\Delta \rho^{2} \geq 2(n-1) k r \cot (k r)+2, \quad \text { if } \kappa(q, r)=k^{2}, r<\frac{\pi}{2 k},
\end{aligned}
$$

and

$$
\operatorname{div} X=\Delta \rho^{2} \geq 2(n-1) k r \operatorname{coth}(k r)+2, \quad \text { if } \kappa(q, r)=-k^{2},
$$

which implies

$$
\begin{gathered}
c\left(B_{M}(q, r)\right) \geq \frac{n}{r}, \quad \text { if } \kappa(q, r)=0, \\
c\left(B_{M}(q, r)\right) \geq \frac{(n-1) k r \cot (k r)+1}{r}, \quad \text { if } \kappa(q, r)=k^{2}, r<\frac{\pi}{2 k},
\end{gathered}
$$

and

$$
c\left(B_{M}(q, r)\right) \geq \frac{(n-1) k r \operatorname{coth}(k r)+1}{r}, \quad \text { if } \kappa(q, r)=-k^{2} .
$$

By applying Lemma 1, one can obtain estimates in Theorem 5. However, as pointed out in Remark 2, in order to use estimates in Lemma 1, one has to show $\int_{B_{M}(q, r)} \operatorname{div}\left(|f|^{p} X\right)=0$ or $\int_{B_{M}(q, r)} \operatorname{div}\left(f^{2} X e^{-\varphi}\right)=0$ for all $f \in$ $C_{0}^{\infty}\left(B_{M}(q, r)\right)$ and the chosen vector filed $X$ which is smooth almost everywhere in $B_{M}(q, r)$. This fact can be easily proven through replacing $\operatorname{div}\left(f^{2} X\right)$ by $\operatorname{div}\left(|f|^{2} X e^{-\varphi}\right)$ or $\operatorname{div}\left(|f|^{p} X\right)$ in the last part of the proof of [1, Theorem 4.1].

REMARK 10. If $\kappa(q, r)=-k^{2}$ or $\kappa(q, r)=0$, then $\operatorname{inj}(q)=\infty$, which implies that $M$ is noncompact. For the case of $\kappa(q, r)=-k^{2}$, letting $r \rightarrow \infty$, then $B_{M}(B(q, r))$ tends to $M$, and $\lambda_{1, \varphi}(M) \geq\left[\frac{(n-1) k-c^{+}}{2}\right]^{2}$ and $\lambda_{1, p}(M) \geq$ $\left[\frac{(n-1) k}{p}\right]^{p}$, which are exactly the estimates given in Lemma 2 . If $\varphi=$ const. (or $p=2$ ) and $M$ has non-positive sectional curvature (which satisfies assumption $\kappa(q, r)=0)$, then $\lambda_{1, \varphi}\left(B_{M}(q, r)\right)=\lambda_{1}\left(B_{M}(q, r)\right) \quad\left(\right.$ or $\lambda_{1, p}\left(B_{M}(q, r)\right)=$ $\left.\lambda_{1}\left(B_{M}(q, r)\right)\right)$ and by Theorem 5 , one has $\lambda_{1}\left(B_{M}(q, r)\right) \geq \frac{n^{2}}{4 r^{2}}$, which is not so good as the estimate (24), since $J_{n / 2-1}>\frac{n}{2}$ for $n \in \mathbb{N}_{+}$and $n \geq 2$. However, this lower bound becomes more and more sharper as $n$ increases, since $2 J_{n / 2-1} / n \rightarrow 1$ as $n \rightarrow \infty$. 


\section{Acknowledgement}

The authors would like to thank the referee for his or her careful reading and interesting comments such that the paper appears as its present version.

\section{References}

[1] G.-P. Bessa and J.-F. Montenegro, Eigenvalue estimates for submanifolds with locally bounded mean curvature, Ann. Glob. Anal. Geom., 24(2) (2003), 279-290.

[2] G.-P. Bessa and J.-F. Montenegro, An extension of Barta's theorem and geometric applications, Ann. Glob. Anal. Geom., 31(4) (2007), 345-362.

[3] G.-P. Bessa and M.-S. Costa, Eigenvalue estimates for submanifolds with locally bounded mean curvature in $N \times R$, Proc. Amer. Math. Soc., 137 (2009), 1093-1102.

[4] I. Chavel, Eigenvalues in Riemannian Geometry, Academic Press, New York, 1984.

[5] S.-Y. Cheng, Eigenfunctions and eigenvalues of the Laplacian, Am. Math. Soc. Proc. Symp. Pure Math., 27 (Part II) (1975), 185-193.

[6] L. F. Cheung and P. F. Leung, Eigenvalue estimates for submanifolds with bounded mean curvature in the hyperbolic space, Math. Z., 31 (2001), 525-530.

[7] F. Du and J. Mao, Reilly-type inequalities for the $p$-Laplacian on compact Riemannian manifolds, Front. Math. China, 10 (2015), 583-594.

[8] F. Du and J. Mao, Estimates for the first eigenvalue of the drifting Laplace and the $p$-Laplace operators on submanifolds with bounded mean curvature in the hyperbolic space, J. Math. Anal. Appl., 456 (2017), 787-795.

[9] L.-B. Hou and J. Mao, The principle of numerical calculations for eigenvalue comparison on parameterized surfaces, J. Math. Research Appl., 38 (2018), 58-62.

[10] P. Freitas, J. Mao and I. Salavessa, Spherical symmetrization and the first eigenvalue of geodesic disks on manifolds, Calc. Var. Partial Differential Equations, 51 (2014), $701-724$.

[11] A. Grigor'yan, Analytic and geometric background of recurrence and non-explosion of the Brownian motion on Riemannian manifolds, Bull. Amer. Math. Soc., 36(2) (1999), 135-249.

[12] H.-Z. Li and Y. Wei, $f$-minimal surface and manifold with positive $m$-Bakry-Émery Ricci curvature, J. Geom. Anal., 25 (2015), 421-435.

[13] J. Mao, Eigenvalue estimation and some results on finite topological type, Ph.D. thesis, IST-UTL, 2013.

[14] J. Mao, Eigenvalue inequalities for the $p$-Laplacian on a Riemannian manifold and estimates for the heat kernel, J. Math. Pures Appl., 101(3) (2014), 372-393.

[15] W. Meeks and H. Rosenberg, Stable minimal surfaces in $M^{2} \times \mathbb{R}$, J. Differential Geom., 68(3) (2004), 515-534.

[16] W. Meeks and H. Rosenberg, The theory of minimal surfaces in $M^{2} \times \mathbb{R}$, Comment. Math. Helv., 80(4) (2005), 811-858.

[17] H.-P. Mckean, An upper bound for the spectrum of $\Delta$ on a manifold of negative curvature, J. Differential Geom., 4 (1970), 359-366.

[18] R. Schoen and S.-T. Yau, Lectures on Differential Geometry, International Press, Boston, 1994. 
[19] G. Wei and W. Wylie, Comparison geometry for the Bakry-Émery Ricci tensor, J. Differential Geom., 83 (2009), 377-405.

\section{Jing Mao}

Faculty of Mathematics and Statistics

Key Laboratory of Applied Mathematics of Hubei Province

Hubei University, Wuhan, 430062, CHINA

E-mail: jiner120@163.com

URL: http://202.114.144.124/info/1057/1105.htm

\section{Rongqiang Tu}

Faculty of Mathematics and Statistics

Hubei University, Wuhan, 430062, CHINA

Kai Zeng

Faculty of Mathematics and Statistics

Hubei University, Wuhan, 430062, CHINA 